OPEN ACCESS

Edited by:

Matteo Ferro,

European Institute of Oncology (IEO),

Italy

Reviewed by:

Francesco Soria,

University of Turin, Italy

Anca lleana Sin,

Pharmacy, Sciences and Technology

of Târgu Mureş, Romania

*Correspondence:

Mateusz Adamkiewicz

uro.adamkiewicz@gmail.com

Specialty section:

This article was submitted to

Genitourinary Oncology,

a section of the journal

Frontiers in Oncology

Received: 18 January 2021 Accepted: 09 March 2021

Published: 26 March 2021

Citation:

Adamkiewicz M, Bryniarski P,

Kowalik M, Burzyński B, Rajwa $P$ and Paradysz A (2021) Lymphocyte-toMonocyte Ratio Is the Independent Prognostic Marker of Progression in

Patients Undergoing BCGImmunotherapy for Bladder Cancer.

Front. Oncol. 11:655000.

doi: 10.3389/fonc.2021.655000

\section{Lymphocyte-to-Monocyte Ratio Is the Independent Prognostic Marker of Progression in Patients Undergoing BCG-Immunotherapy for Bladder Cancer}

\author{
Mateusz Adamkiewicz ${ }^{1 *}$, Piotr Bryniarski ${ }^{1}$, Maksymilian Kowalik ${ }^{1}$, Barttomiej Burzyński ${ }^{2}$, \\ Pawet Rajwa ${ }^{1}$ and Andrzej Paradysz ${ }^{1}$ \\ 1 Department of Urology, Medical University of Silesia, Zabrze, Poland, ${ }^{2}$ Department of Rehabilitation, Faculty of Health \\ Sciences, Medical University of Silesia, Katowice, Poland
}

Introduction: Transurethral resection of bladder tumor with subsequent BCG immunotherapy is the current gold standard in the treatment of high risk and some mediumrisk non-muscle invasive bladder cancer. Clinical factors like stage, grade, age and gender are well-know predictors of progression to muscle-invasive bladder cancer. In recent years novel hematological biomarkers were shown to be independent predictors of progression. This study aimed to evaluate which of these novel markers has the highest prognostic value of progression in patients with bladder cancer receiving BCG immunotherapy.

Materials and methods: We retrospectively analyzed the data of 125 patients with nonmuscle invasive bladder cancer who received BCG immunotherapy. Of these, 61 progressed to muscle-invasive disease or had high-grade recurrence. These patients were compared with the group who did not progress $(n=64)$. Clinical data including stage, grade, age, gender, smoking status and observational time was collected. Besides, information on blood count analysis was obtained from ambulatory digital charts. On this basis neutrophil-to-lymphocyte ratio (NLR), platelet-to lymphocyte ratio (PLR) and lymphocyte-to-monocyte ratio (LMR) was counted and compared between groups.

Results: NLR, PLR and LMR were shown to be independent prognostic markers of progression in multivariable analysis. The model with stage, grade, age, gender, smoking status and LMR had the highest prognostic values of all models (area under curve $[A \cup C]=$ 0.756). The cut-off point according to ROC curves for LMR was 3.25. Adding LMR to the baseline model including clinical variables significantly increased area under curve by $0.08(p=$ 0.001). NLR and PLR did not increase areas under curve significantly to baseline model.

Conclusions: LMR outperformed NLR and PLR for prediction of progression in patients with non-muscle-invasive bladder cancer receiving BCG immunotherapy. LMR, as an easily obtainable biomarker, should be incorporated to the present risk stratification models.

Keywords: lymphocyte-to-monocyte ratio, neutrophil-to-lymphocyte ratio, platelet-to-lymphocyte ratio, bladder cancer, transurethral resection of bladder tumor, Bacillus Calmet-Guiren immunotherapy 


\section{INTRODUCTION}

Bladder cancer ( $\mathrm{BCa})$ is the ninth most common cancer worldwide (1). Most of the patients are initially diagnosed with non-muscle invasive $\mathrm{BCa}$ (NMIBC), but up to $45 \%$ will progress to muscle-invasive disease within 5 years $(2,3)$. The goal of NMIBC bladder-sparing management is to achieve local control of the disease, and defer or avoid radical cystectomy. At present, the most common adjuvant treatment in intermediate and highrisk patients includes BCG (Bacillus Calmette-Guerin) immunotherapy. Present evidence suggest its efficacy with a $32 \%$ decrease in the recurrence rate and $27 \%$ in progression rate to muscle-invasive disease (4). It comprises of induction and maintenance phases, lasting up to three years. According to recent evidence, the efficacy of such therapy is based on local and systemic immunological responses $(5,6)$.

Despite advances in NMIBC management there is a lack of reliable biomarkers that could guide follow-up after TURBT and BCG instillations. Blood-based inflammatory biomarkers such as neutrophil-to-lymphocyte ratio (NLR), platelet-to-lymphocyte ratio (PLR) were introduced as a valuable prognostic markers for urologic cancers, including bladder cancer (7-14), Recent evidence indicates that a lower peripheral lymphocyte-to-monocyte ratio (LMR) is closely associated with worse prognosis in patients with various cancers and could be considered as another easily available and reliable prognostic biomarker $(9,15,16)$.

Improvement in risk stratification of NMIBC is particularly important, as could lead to personalized surveillance tailoring, and delineation of disease trajectory, which would result in patient's quality of life improvement, cost-effectiveness and enhanced oncological outcomes. Therefore, our primary goal was to evaluate and compare the prognostic role of NLR, PLR and LMR in a cohort of patients who underwent BCGimmunotherapy for NMIBC.

\section{MATERIAL AND METHODS}

We retrospectively analyzed the data from our ambulatory and department digital charts of patients with NMIBC who underwent TURBT followed by BCG immunotherapy. Inclusion criteria were as follows: initial BCG therapy starting not earlier than 6 years ago, complete data regarding all pathology reports, complete follow-up cystoscopy and subsequent treatment after recurrence and progression during BCG therapy. We also obtained the information if a patient eventually died due to bladder cancer. We considered patients with medium-risk recurrent low-grade non-invasive (pTa) disease as patients without progression. We excluded patients with sole carcinoma in-situ (only 12 patients) and those intolerant for BCG therapy. The group of patients with both pT1 and Carcinoma in-situ (CIS) (the highest risk group) was combined with pT1 group as only seven patients in this group received BCG therapy (most of these patients underwent early cystectomy). Progression was defined as the disease changing its pathology from non-muscle invasive to muscle-invasive or the recurrence of high-grade disease. Some 173 patients met these criteria, and 61 of these progressed. With propensity score matching we paired each patient with progression to another patient without progression according to age and gender. For three pairs we found additional patients without progression with almost the same propensity score and they were also included in the analysis.

Finally, the database of 125 patients (61 with progression and 64 without progression) was analyzed. All patients received Onco-BCG according to Morales scheme. Follow-up consisted of cystoscopy, cytology, and contrast enhanced computer tomography in time intervals according to EAU guidelines.

\section{Statistical Analysis}

Demographic variables were compared with t-test for continuous variables and Chi-square test for categorical variables. In the case of skewed distributions of data logarithmic transformation was applied to correct for normality and stabilize the variance. We constructed four models based on logistic regression. The first model (with only clinical data) counted the probabilities of each patient for developing progression based on stage, grade, age, gender and smoking status. Subsequent three models comprised the clinical data and one hematological variable. The second model consisted of clinical data and LMR, third model-clinical data and PLR, fourth model clinical data and NLR. Finally, the models nos. 2, 3 and 4 were compared with the baseline model (no. 1) in ROC curves. We considered patients with longer observational times and those who eventually died of bladder cancer more important to our analysis. For this analysis we applied weights to each patient equal to observational times (which started on the day of first BCG dose and ending: on the December of 2020 for those without progression; on the day of progression for those with progression). The highest weight was given to patients who eventually died due to bladder cancer. In addition we analyzed the impact of clinical and hematological variables on progression free survival with Cox regression. Similarly to four logistic regression models we constructed the same four models but analyzed them with Cox regression. Harrell's C-index for each model was counted. All data were analyzed with Statistica Statsoft package ver 13. P values of less than $5 \%$ were considered statistically significant.

\section{RESULTS}

Demographic and clinical characteristics are shown in Table 1. As patients were matched according to propensity score counted based on age and gender these two are not different between groups. Table 2 shows the outcomes of logistic regression with clinical data and LMR (Model no. 2). Odd ratios (OR) show how unfavorable factors worsen the chance of developing progression. For example, patients with pT1 have over four times higher odds for developing progression than patients with pTa. On the other hand, continuous data show the increase in odds when changing its value of one unit. If the value of OR is lower than 1 (like LMR) the odds decrease. 
Therefore, the increase in LMR of 1 decreases the odds of developing progression of $46 \%((1-0.54) * 100 \%)$.

Figure 1 shows ROC curves for models 1 and 2 increase in area under curve (AUC) after adding LMR to the model no. 1 is about 0.08 and this value is statistically significant $(p=0.001)$. Model numbers 3 and 4 show that NLR and PLR respectively did not increase significantly prognostic values of baseline models (Figures 2 and 3).

From all hematological biomarkers, LMR showed the highest prognostic value in the prediction of bladder cancer progression in patients receiving BCG immunotherapy. Based on the Youden index, the optimal cut off point of LMR was 3.25, with reverse association with progression (Figure 4). Table 3 shows our group of patients stratified by that cut-off point.

Stage $(\mathrm{HR}=2.26 ; \mathrm{p}=0.04)$, grade $(\mathrm{HR}=1.42 ; \mathrm{p}<0.01)$ and smoking status $(\mathrm{HR}=1.13 ; \mathrm{p}<0.01)$ had significant impact on progression free survival in model 1 . In models 2,3 and 4 all clinical variables remained significant. LMR in model 2, PLR in model 3 and NLR in model 4 were also significant with $H R=0.72(\mathrm{p}<0.01)$, 1.006 ( $\mathrm{p}<0.01)$ and 1.13 ( $\mathrm{p}<0.01)$, respectively. Harrell's C-indices for models $1-4$ were: $0.731 ; 0.783 ; 0.738$ and 0.741 respectively.

\section{DISCUSSION}

In 2009, Spanish Urological Club for Oncological Treatment (CUETO) developed a risk stratification model predicting progression which was based on clinical data for patients with non-muscle invasive bladder cancer receiving $B C G$ immunotherapy (17). However, despite broad implementation, it still lacks of sufficient accuracy. Our study not only focuses on the prognostic value of the included hematological biomarkers but also assesses the added value of each one. It is quite clear that all markers improve, to some extent, the prognostic strength of contemporary models (e.g., CUETO) but our study indicates that among all markers LMR has the highest added value.

In recent years it was shown that not only clinical parameters like stage, grade, presence of carcinoma in situ, age, smoking status and gender are predictors of bladder cancer progression but others also play an important role. Among them are hematological biomarkers like LMR, NLR, PLR, derived neutrophil-tolymphocyte ratio (dLNR) or even comorbidities, such as diabetes $(18,19)$. The most studied biomarkers is NLR which showed the prognostic value for invasiveness and progression of bladder cancer, albeit with varying accuracy (20-26). Albayrak et al. emphasized the fact that after adjusting for age NLR lost its prognostic value (27). NLR also predicted progression and recurrence in patients with bladder cancer receiving BCG immunotherapy $(13,28)$. Yuk et al. showed that in patients receiving BCG immunotherapy NLR was a good predictor of overall survival but not progression-free survival (29).

PLR on the other hand did not show the prognostic value for invasiveness of bladder cancer (20) but the recent meta-analysis underlined its prognostic value for overall survival (30). In patients receiving BCG immunotherapy PLR was not shown to impact progression-free survival (29). Akan et al. showed that PLR predicted progression to muscle invasive disease in patients receiving BCG therapy (31).

TABLE 1 | Demographic and clinical data of patients in analysis.

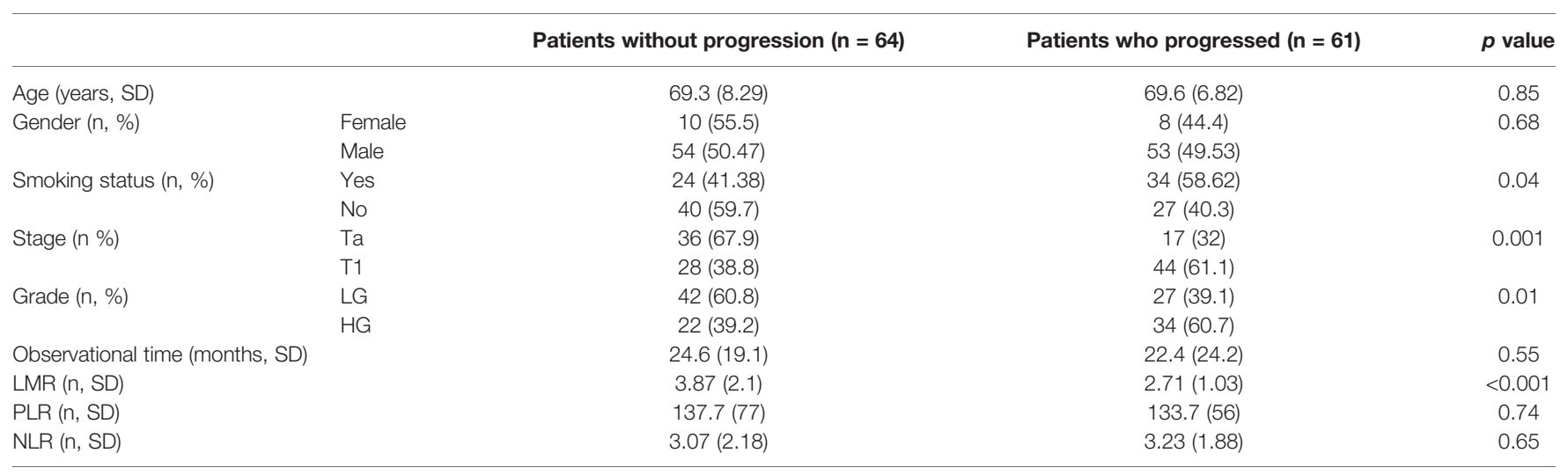

LMR, lymphocyte to monocyte ratio; PLR, platelet to lymphocyte ratio; NLR, neutrophil to lymphocyte ratio.

TABLE 2 | Multivariate analysis of second model.

\begin{tabular}{|c|c|c|c|c|c|}
\hline & Variable & OR & $\mathrm{Cl}(+95 \%)$ & Cl (-95\%) & $p$ value \\
\hline LMR & & 0.546 & 0.506 & 0.590 & 0.000 \\
\hline Grade & $\mathrm{HG}$ & 3.205 & 2.609 & 3.937 & 0.000 \\
\hline Gender & Male & 1.059 & 0.802 & 1.397 & 0.683 \\
\hline Smoking status & Yes & 2.177 & 1.822 & 2.601 & 0.000 \\
\hline
\end{tabular}

Odds ratios refer to: pT1 vs pTa; HG vs LG; male vs female; smokers vs non-smokers. Cl, confidence interval; OR, Odds ratio; LMR, lymphocyte-to-monocyte ratio. 


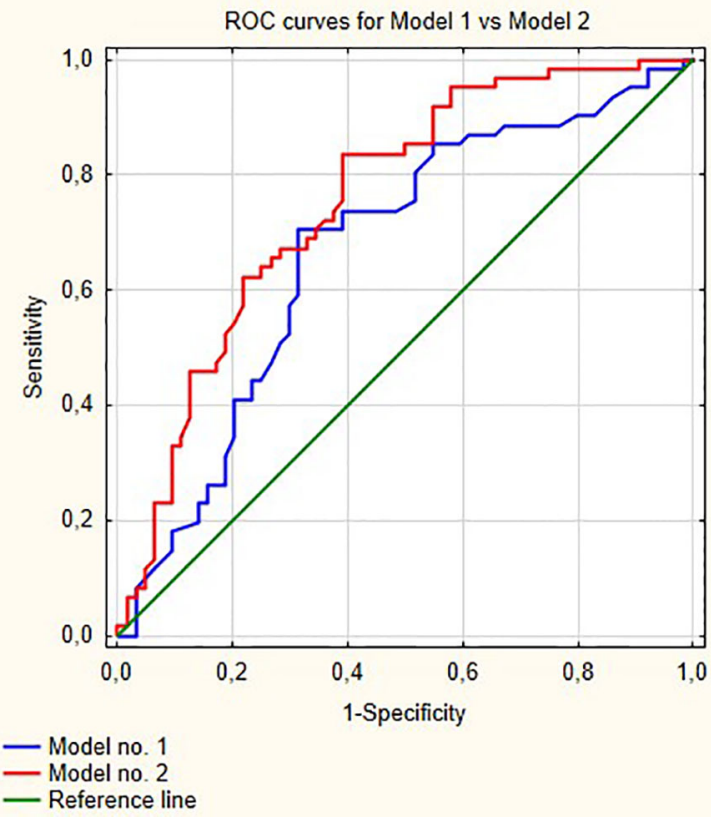

FIGURE 1 | Comparisons of ROC curves for models 1 and 2. AUC for model $1-0.670$; model $2-0.756, p=0.001$.

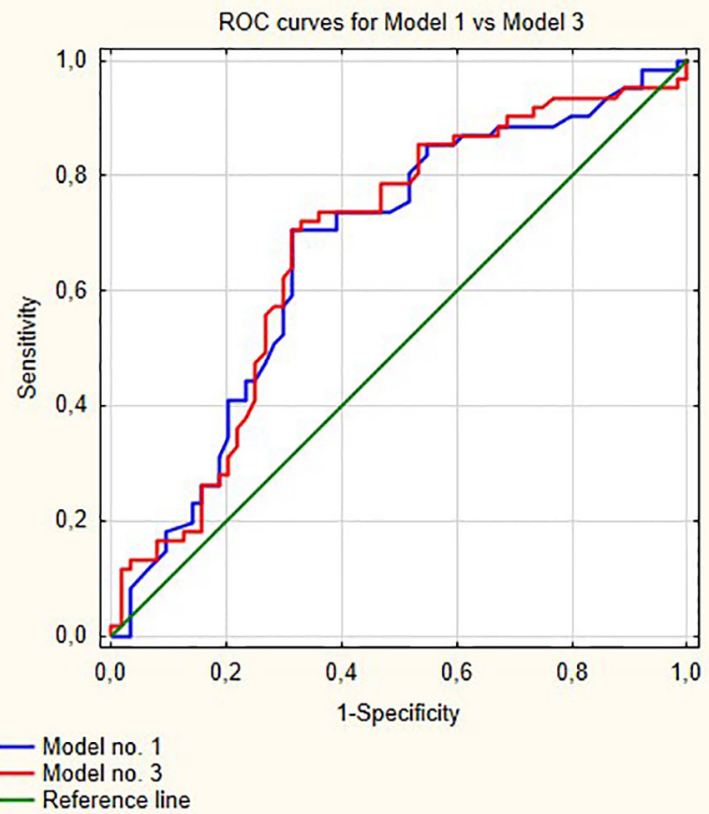

FIGURE 2 | Comparisons of ROC curves for models 1 and 3. AUC for model $1-0.67$; model $2-0.677, p=0.53$.

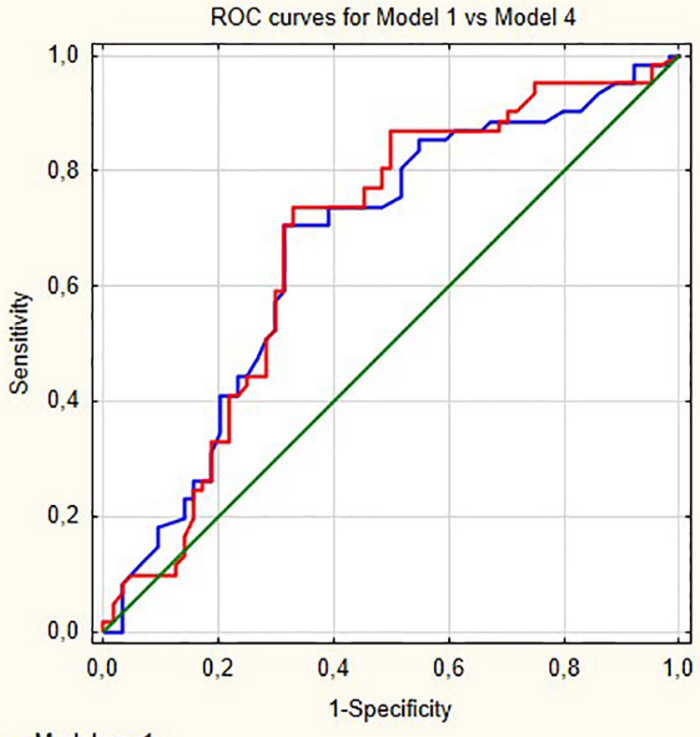

- Model no. 1

- Model no. 4

- Reference line

FIGURE 3 | Comparison of ROC curves for models 1 and 4 . AUC for model $1-0.670$; model $2-0.676, p=0.58$.

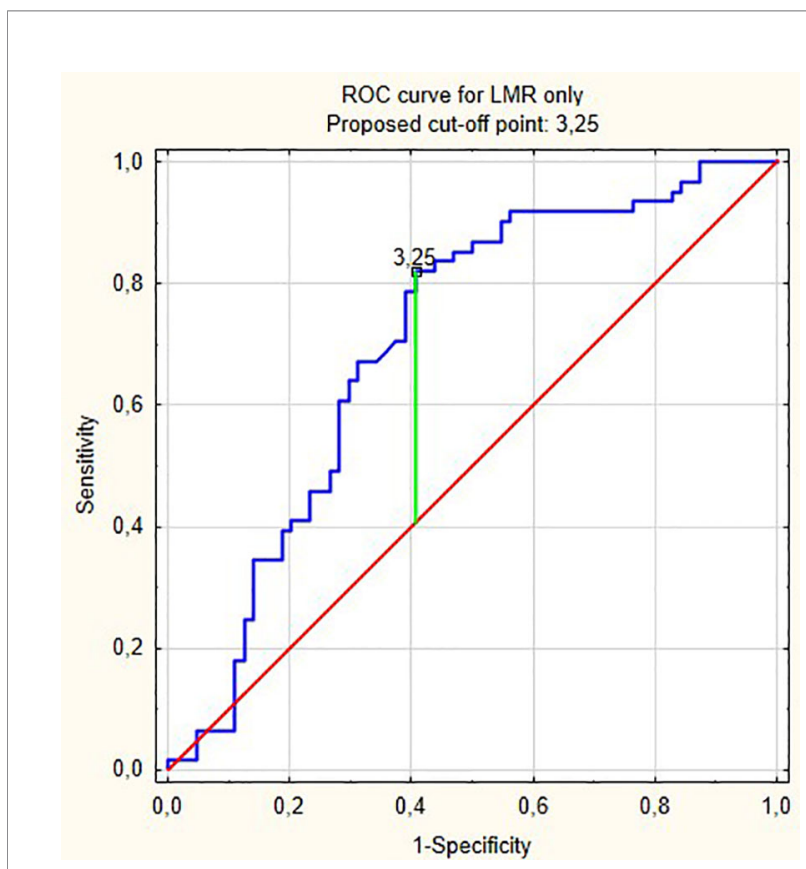

FIGURE 4 | ROC curve for LMR only. Proposed cut-off point: 3,25. 
TABLE 3 | Clinical parameters in patients divided by LMR cut-off point.

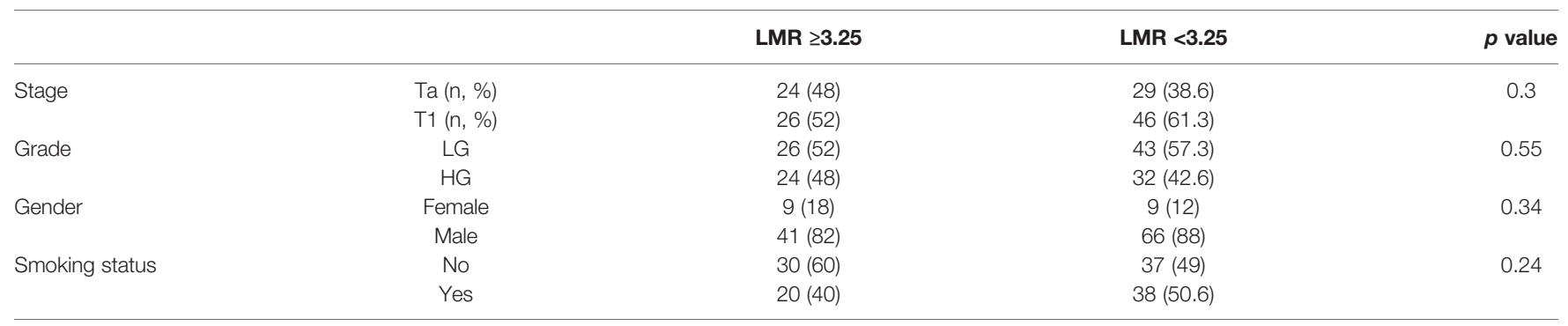

LMR was also found as a good prognostic marker for survival in a recent meta-analysis in BCa patients undergoing radical cystectomy (9). Some authors indicate its superiority over NLR in prognostic strength of unfavorable course of disease (16). However, the prognostic value of LMR has not yet been studied in NMIBC patients receiving BCG immunotherapy.

In our multivariable analysis after adjusting for age, stage, grade, gender and smoking status LMR $(\mathrm{OR}=0.54 ; \mathrm{p}<0.001)$, $\operatorname{PLR}(\mathrm{OR}=1.001 ; \mathrm{p}=0.04)$ and NLR $(\mathrm{OR}=1.05 ; \mathrm{p}=0.02)$ were independent prognostic factors for progression in model nos. 2, 3 and 4, respectively. However, only LMR increased significantly the prognostic value of the model (Figure 1) indicating that this variable explains part of the variance which is not elucidated by clinical variables in the baseline model. PLR and NLR also explained such variance but the difference was not significant (Figures 2 and 3). According to information in Table 3 LMR does not correlate with preoperative clinical data and thus explains propensity of developing progression independently.

Despite its pioneer nature, our study has several limitations. First, this is a single-center, retrospective study including only Caucasian patients. Second, we did not adjust the model for the effect of possible other comorbidities or drugs, which could influence biomarkers values. Despite these, we present the first study which determined LMR as independent and the most prognostic factor for progression in NMIBC treated with BCG-immunotherapy.

\section{CONCLUSION}

LMR is an independent predictor of progression in patients with NMIBC receiving BCG immunotherapy. Its prognostic strength

\section{REFERENCES}

1. Antoni S, Ferlay J, Soerjomataram I, Znaor A, Jemal A, Bray F. Bladder Cancer Incidence and Mortality: A Global Overview and Recent Trends. Eur Urol (2017) 71(1):96-108. doi: 10.1016/j.eururo.2016.06.010

2. Sylvester RJ, Van Der Meijden APM, Oosterlinck W, Witjes JA, Bouffioux C, Denis L, et al. Predicting recurrence and progression in individual patients with stage Ta T1 bladder cancer using EORTC risk tables: A combined analysis of 2596 patients from seven EORTC trials. Eur Urol (2006) 49 (3):466-77. doi: 10.1016/j.eururo.2005.12.031

3. van Rhijn BWG, Burger M, Lotan Y, Solsona E, Stief CG, Sylvester RJ, et al. Recurrence and Progression of Disease in Non-Muscle-Invasive Bladder surpasses NLR and PLR in the prediction of progression in patients with bladder cancer receiving BCG immunotherapy.

\section{DATA AVAILABILITY STATEMENT}

The raw data supporting the conclusions of this article will be made available by the authors, without undue reservation.

\section{ETHICS STATEMENT}

Ethical review and approval was not required for the study on human participants in accordance with the local legislation and institutional requirements. The patients/participants provided their written informed consent to participate in this study.

\section{AUTHOR CONTRIBUTIONS}

MA and PR designed the study. MA and PB wrote the paper with input from all authors. $\mathrm{PB}$ derived the models and analyzed the data. MA, MK, and BB gathered the data for statistical purposes. AP offered mentorship on every part of the research. All authors contributed to the article and approved the submitted version.

\section{FUNDING}

This publication was funded by Medical University of Silesia, Katowice, Poland.

Cancer: From Epidemiology to Treatment Strategy. Eur Urol (2009) 56 (3):430-42. doi: 10.1016/j.eururo.2009.06.028

4. Babjuk M, Burger M, Compérat EM, Gontero P, Mostafid AH, Palou J, et al. European association of urology guidelines on non-muscle-invasive bladder cancer (TaT1 and carcinoma In Situ) - 2019 Update. Eur Urol (2019). doi 10.1016/j.eururo.2019.08.016

5. Pettenati C, Ingersoll MA. Mechanisms of BCG immunotherapy and its outlook for bladder cancer. Nat Rev Urol (2018) 15(10):615-25. doi: 10.1038/ s41585-018-0055-4

6. Schamhart DH, Kurth KH, de Reijke TM, Vleeming R. BCG treatment and the importance of an inflammatory response. Urol Res (1992) 20(3):199-203. doi: $10.1007 / \mathrm{BF} 00299717$ 
7. Lee SM, Russell A, Hellawell G. Predictive value of pretreatment inflammation-based prognostic scores (Neutrophil-to-lymphocyte ratio, platelet-to-lymphocyte ratio, and lymphocyte-to-monocyte ratio) for invasive bladder carcinoma. Korean J Urol (2015) 56(11):749-55. doi: 10.4111/kju.2015.56.11.749

8. Lucca I, Hofbauer SL, Leitner CV, de Martino M, Özsoy M, Susani M, et al. Development of a Preoperative Nomogram Incorporating Biomarkers of Systemic Inflammatory Response to Predict Nonorgan-confined Urothelial Carcinoma of the Bladder at Radical Cystectomy. Urology (2016) 95:132-8. doi: 10.1016/j.urology.2016.06.007

9. Ma JY, Hu G, Liu Q. Prognostic significance of the lymphocyte-to-monocyte ratio in bladder cancer undergoing radical cystectomy: A meta-analysis of 5638 individuals. Dis Markers (2019) 2019:1-8. doi: 10.1155/2019/7593560

10. Rajwa P, Życzkowski M, Paradysz A, Slabon-Turska M, Suliga K, Bujak K, et al. Novel hematological biomarkers predict survival in renal cell carcinoma patients treated with nephrectomy. Arch Med Sci (2017) 16(5):1062-71. doi: 10.5114/aoms.2017.70250

11. Vartolomei MD, Ferro M, Cantiello F, Lucarelli G, Di Stasi S, Hurle R, et al. Validation of Neutrophil-to-lymphocyte Ratio in a Multi-institutional Cohort of Patients With T1G3 Non-muscle-invasive Bladder Cancer. Clin Genitourin Cancer (2018) 16(6):445-52. doi: 10.1016/j.clgc.2018.07.003

12. Ferro M, Di Lorenzo G, Vartolomei MD, Bruzzese D, Cantiello F, Lucarelli G, et al. Absolute basophil count is associated with time to recurrence in patients with high-grade T1 bladder cancer receiving bacillus Calmette-Guérin after transurethral resection of the bladder tumor. World J Urol (2019) 38(1):14350. doi: 10.1007/s00345-019-02754-2 (0123456789).

13. Vartolomei MD, Porav-Hodade D, Ferro M, Mathieu R, Abufaraj M, Foerster B, et al. Prognostic role of pretreatment neutrophil-to-lymphocyte ratio (NLR) in patients with non-muscle-invasive bladder cancer (NMIBC): A systematic review and meta-analysis. Urol Oncol Semin Orig Investig (2018) 36 (9):389-99. doi: 10.1016/j.urolonc.2018.05.014

14. Vartolomei MD, Kimura S, Ferro M, Vartolomei L, Foerster B, Abufaraj M, et al. Is neutrophil-to-lymphocytes ratio a clinical relevant preoperative biomarker in upper tract urothelial carcinoma? A meta-analysis of 4385 patients. World J Urol (2018) 36(7):1019-29. doi: 10.1007/s00345-018-2235-5

15. Li J, Cheng Y, Ji Z. Prognostic value of pretreatment lymphocyte-to-monocyte ratio in patients with urologic tumors: A PRISMA-compliant meta-analysis. Med (Baltimore) (2019) 98(2):e14091. doi: 10.1097/MD.0000000000014091

16. Yoshida T, Kinoshita H, Yoshida K, Mishima T, Yanishi M, Inui H, et al. Prognostic impact of perioperative lymphocyte-monocyte ratio in patients with bladder cancer undergoing radical cystectomy. Tumor Biol (2016) 37 (8):10067-74. doi: 10.1007/s13277-016-4874-8

17. Fernandez-Gomez J, Madero R, Solsona E, Unda M, Martinez-Piñeiro L, Gonzalez M, et al. Predicting Nonmuscle Invasive Bladder Cancer Recurrence and Progression in Patients Treated With Bacillus Calmette-Guerin: The CUETO Scoring Model. J Urol (2009) 182(5):2195-203. doi: 10.1016/ j.juro.2009.07.016

18. Rajwa P, Życzkowski M, Paradysz A, Bujak K, Bryniarski P. Evaluation of the prognostic value of LMR, PLR, NLR, and dNLR in urothelial bladder cancer patients treated with radical cystectomy. Eur Rev Med Pharmacol Sci (2018) doi: 10.26355/eurrev_201805_15060

19. Ferro M, Katalin MO, Buonerba C, Marian R, Cantiello F, Musi G, et al. Type 2 diabetes mellitus predicts worse outcomes in patients with high-grade T1 bladder cancer receiving bacillus Calmette-Guérin after transurethral resection of the bladder tumor. Urol Oncol Semin Orig Investig (2020) 38 (5):459-64. doi: 10.1016/j.urolonc.2020.02.016

20. Kaynar M, Yıldırım ME, Badem H, Çaviş M, Tekinarslan E, İstanbulluoğlu $\mathrm{MO}$, et al. Bladder cancer invasion predictability based on preoperative neutrophil-lymphocyte ratio. Tumor Biol (2014) 35(7):6601-5. doi: 10.1007/ s13277-014-1889-x

21. Lucca I, Jichlinski P, Shariat SF, Rouprêt M, Rieken M, Kluth LA, et al. The Neutrophil-to-lymphocyte Ratio as a Prognostic Factor for Patients with Urothelial Carcinoma of the Bladder Following Radical Cystectomy: Validation and Meta-analysis. Eur Urol Focus (2016) 2(1):79-85. doi: 10.1016/j.euf.2015.03.001

22. D’Andrea D, Moschini M, Gust K, Abufaraj M, Özsoy M, Mathieu R, et al. Prognostic Role of Neutrophil-to-Lymphocyte Ratio in Primary Non-muscleinvasive Bladder Cancer. Clin Genitourin Cancer (2017) 15(5):e755-64. doi: 10.1016/j.clgc.2017.03.007

23. Hermanns T, Bhindi B, Wei Y, Yu J, Noon AP, Richard PO, et al. Pretreatment neutrophil-to-lymphocyte ratio as predictor of adverse outcomes in patients undergoing radical cystectomy for urothelial carcinoma of the bladder. Br J Cancer (2014) 111(3):444-51. doi: 10.1038/bjc.2014.305

24. Celik O, Akand M, Keskin MZ, Yoldas M, Ilbey YO. Preoperative neutrophil-tolymphocyte ratio (NLR) may be predictive of pathologic stage in patients with Bladder cancer larger than $3 \mathrm{~cm}$. Eur Rev Med Pharmacol Sci (2016) 20(4):652-6.

25. Gondo T, Nakashima J, Ohno Y, Choichiro O, Horiguchi Y, Namiki K, et al. Prognostic value of neutrophil-to-lymphocyte ratio and establishment of novel preoperative risk stratification model in bladder cancer patients treated with radical cystectomy. Urology (2012) 79(5):1085-91. doi: 10.1016/j.urology.2011.11.070

26. Mano R, Baniel J, Shoshany O, Margel D, Bar-On T, Nativ O, et al. Neutrophil-to-lymphocyte ratio predicts progression and recurrence of non-muscle-invasive bladder cancer. Urol Oncol Semin Orig Investig (2015) 33(2):67.e1-7. doi: 10.1016/j.urolonc.2015.05.017

27. Albayrak S, Zengin K, Tanik S, Atar M, Unal SH, Imamoglu MA, et al. Can the neutrophil-to-lymphocyte ratio be used to predict recurrence and progression of non-muscle-invasive bladder cancer? Kaohsiung J Med Sci (2016) 32 (6):327-33. doi: 10.1016/j.kjms.2016.05.001

28. Racioppi M, Di Gianfrancesco L, Ragonese M, Palermo G, Sacco E, Bassi PF. Can Neutrophil-to-Lymphocyte ratio predict the response to BCG in highrisk non muscle invasive bladder cancer? Int Braz J Urol (2019) 45(2):315-24. doi: 10.1590/s1677-5538.ibju.2018.0249

29. Yuk HD, Jeong CW, Kwak C, Kim HH, Ku JH. Elevated neutrophil to lymphocyte ratio predicts poor prognosis in non-muscle invasive bladder cancer patients: Initial intravesical bacillus Calmette-Guerin treatment after transurethral resection of bladder tumor setting. Front Oncol (2019) 9 (JAN):642. doi: 10.3389/fonc.2018.00642

30. Tagawa ST, Singer EA, Anna Mess E, Wang X, Ni X, Tang G. Prognostic Role of Platelet-to-Lymphocyte Ratio in Patients With Bladder Cancer: A MetaAnalysis. Front Oncol (2019) 1:757. doi: 10.14744/etd.2019.74507

31. Akan S, Ediz C, Sahin A, Tavukcu HH, Urkmez A, Horasan A, et al. Can the systemic immune inflammation index be a predictor of BCG response in patients with high-risk non-muscle invasive bladder cancer? Int J Clin Pract (2020) 75(4). doi: 10.1111/ijcp.13813

Conflict of Interest: The authors declare that the research was conducted in the absence of any commercial or financial relationships that could be construed as a potential conflict of interest.

Copyright (๔) 2021 Adamkiewicz, Bryniarski, Kowalik, Burzyński, Rajwa and Paradysz. This is an open-access article distributed under the terms of the Creative Commons Attribution License (CC BY). The use, distribution or reproduction in other forums is permitted, provided the original author(s) and the copyright owner(s) are credited and that the original publication in this journal is cited, in accordance with accepted academic practice. No use, distribution or reproduction is permitted which does not comply with these terms. 